\title{
PROFUNDIDADE DE AMOSTRAGEM DO SOLO PARA AVALIAÇÃO DO ENXOFRE DISPONIVEL EM POMAR DE LIMÃO SICILIANO (Citrus limon Burm.) ${ }^{1}$
}

\author{
L.I. PROCHNOW'; A.E. BOARETTO ${ }^{2}$ \\ 'Departamento de Ciência do Solo, ESALQ/USP, C.P. 9, CEP: 13.418.900- Piracicaba,SP \\ ${ }^{2}$ Centro de Energia Nuclear na Agricultura/USP, C.P. 96, CEP: 13.400-900 - Piractcaba,SP
}

\begin{abstract}
RESUMO: Em um Latossolo Vermelho Escuro, textura média, no qual foram estabelecidos diferentes teores de enxofre, atraves da aplicação de doses crescentes de gesso agricola $(0,1,2$ e 4 t/ha), estudou-se a profundidade adequada para coleta de amostras de terra para avaliação do S-SO ${ }_{4}^{-2}$ "disponível" para o limão siciliano (Curus limon Burm.) enxertado sobre porta enxerto Volkameriano. As quantidades de sulfato no solo, extraídas pelo NH,OAC 0,5 M em HOAc 0,25 M, foram determinadas aos 6, 12 e 24 meses ap6s a aplicacão do produto nas seguintes profundidades: 0-5, 5-10, 10-20, 20-40, 40-80 e 80-120 cm. As quantidades de enxofre nas folhas do limão siciliano foram também determinadas na mesma época. Os dados obtidos e os estudos de correlaçăo que se seguiram demonstraram evidéncias de que o limano siciliano teria, no perfodo considerado, extraido enxofre, principalmente, da camada de 0 a $20 \mathrm{~cm}$ de profindidade, e assim sendo, fornece indicíos de que esta deve ser a camada amostrada para fins da avaliação deste importante elemento nesta cultura.

Descritores: disponibilidade de enxofre, gesso agricola, profundidade de amostragem, limão siciliano, Citrus timon
\end{abstract}

\section{SOIL SAMPLING DEPTH FOR AVAILABLE SULPHUR EVALUATION IN SICILIAN LEMON (Cirus limon Burm.) ORCHARD}

SUMMARY: On a medium-textured Dark-Red Latosol (Typic Hapludox), in which increasing levels of sulfate were established by the application of different rates of phosphogypsum $(0,1,2$ e $4 \mathrm{tha})$, a study was carried out to obtain information about the depth of soil samples collected in order to evaluate the $\mathrm{S}^{-\mathrm{SO}_{4}^{-2}}$ availability for the sicilian lemon (Cirus limon Burm.) grafted on "Volkameriano" rootstocks. Sulfate contents at different depths (0-5, 5-10, 10-20, 20-40, 40-80 and $80-120 \mathrm{~cm}$ ) were determined, by using the NH,OAc 0,5 M in HOAc 0,25 M extractor, 6,12 and 24 months after phosphogypsum application. Sulphur content in the leaves of the lemon plants were also determined at the same dates. Analytical data obtained and the correlation studies that followed, showed that the sicilian lemon extracted sulphur mainly from the layer $0-20 \mathrm{~cm}$ and, therefore, this layer is considered representative for soil sample collection, with the purpose of estimating the available sulphnr for sicilian lemon trees.

Key Wonds: sulfur availability, phosphogypsum, sampling deepness, sicilian lemon, Cltrus limon

\section{INTRODUÇÃO}

Uma agricultura moderna deve empregar tecnologia adequada às condições da área a ser cultivada. No que diz respeito a calagem e adubação, a tecnologia rotineira empregada para definir as doses destes insumos têm sido a análise química de terra. Esta técnica constitui-se no meio básico para a transferência das informações da pesquisa para o agricultor (RAJJ et al., 1985).
A análise de rotina atualmente reali-zada pelos laboratórios do Estado de São Paulo (RAIJ \& QUAGGIO, 1983), já tem definidas as premissas básicas (coleta de amostras, métodos analíticos, calibração) para uma interpretação segu-ra dos resultados obtidos. Entretanto, no que diz respeito ao enxofe, muito embora trate-se de um macronutriente, a avaliação da sua disponilidade para as plantas ainda carece de estudos que possam indicar as premissas básicas que, uma vez empre-

\footnotetext{
1 Parte da dissertação do primeiro autor, apresentada à CENA/USP.
}

Sci. agric., Piracicaba, 52(1):101-106, jan./abr. 1995 
gadas, garantam uma interpretação segura dos resultados analíticos obtidos para o elemento.

Um dos problemas é, justamente, a que profundidade devem ser coletadas as amostras de terra. A sugestão têm sido, normalmente, pela recomendação de coletar amostras em diferentes profundidades, uma vez que, em condições tropicais, o elemento tende a se acumular em camadas mais profundas do solo (KINJO, 1980; HARWARD \& REISENAUER, 1966; BARDSLEY \& KILMER, 1963; RAIJ, 1990).

Alguns trabalhos tem realmente confirmado melhores resultados para a avaliação da disponibilidade de enxofre no solo para as plantas, quando a profundidade de coleta foi além dos primeiros 20 cm (BLAKEMORE et al., 1969; BARDSLEY \& KILMER, 1963). Entretanto, estes resultados não devem ser generalizados para todas os casos, pois, dependendo do solo e do sistema radicular do vegetal, podem ocorrer situações em que não é possivel absorver o enxofre das camadas subsuperficiais.

Este estudo teve o objetivo de verificar a profundidade, da qual uma cultura perene, no caso o limão siciliano, absorve a maior parte do enxofre encontrado em suas folhas, e assim, inferir sobre a profundidade de coleta de amostras de terra para fins de avaliação da disponibilidade do elemento nesta cultura.

\section{MATERIAL E MÉTODOS}

Através da adição de doses crescentes $(0,1$, 2 e 4 t/ha) de gesso agrícola (150 $\mathrm{g} / \mathrm{kg}$ de $\mathrm{S}$ e $270 \mathrm{~g} / \mathrm{kg}$ de $\mathrm{CaO}$ ) diferentes teores de enxofre foram estabelecidos (nove repetições delineamento inteiramente casualizado) em um Latossolo Vermelho Escuro da região de BotucatuSP (atributos químicos na TABELA 1) cultivado com limão siciliano (Citrus limon Burm.), porta enxerto Volkameriano.

O pomar, por ocasião da implantacão do experimento (julho de 1988), tinha idade aproximada de 8 anos, com espaçamento de $8 \times 6$ metros.

Amostras compostas de solo foram coletadas, em todas as parcelas, na projeção da copa das plantas, e nas profundidades de $0-5,5-10$, $10-20,20-40,40-80$ e $80-120 \mathrm{~cm}$, aos 6,12 e 24 meses após a aplicação do gesso agrícola. Nestas, após secagem ao ar, foram realizadas as determinações de enxofre "disponível" utilizando-se para tanto do extrator $\mathrm{NH}_{4} \mathrm{OAc} 0,5 \mathrm{M}$ em HOAc 0,25 M (BARDSLEY \& LANCASTER, 1960), na relação terra: extrator de 1 para $2,5\left(10 \mathrm{~cm}^{3} \mathrm{de}\right.$ terra e $25 \mathrm{~mL}$ da solução extratora), empregandose $0,25 \mathrm{~g}$ de carvão ativado por amostra.

A quantificação do enxofre extraído foi realizada por turbidimetria (BARDSLEY \& LANCASTER, 1965).

Amostras de folhas das plantas (terceira ou quarta a partir do fruto, da altura mediana da copa) foram coletadas por ocasião da amostragem do solo. Estas, após secas e moídas, foram submetidas às determinações dos teores de enxofre, com extração nitro-perclórica e quantificação por turbidimetria.

Tabela 1 - Resultados da análise química realizada antes da instalacao do experimento(*).

\begin{tabular}{|c|c|c|c|c|c|c|c|c|c|c|c|}
\hline $\begin{array}{l}\text { Profundidade } \\
\mathrm{cm}\end{array}$ & $\begin{array}{l}\text { P.resina } \\
\mathrm{mg} / \mathrm{dm}^{3}\end{array}$ & $\begin{array}{l}\text { M.O. } \\
\text { g/dm }\end{array}$ & $\begin{array}{r}\mathrm{pH} \\
\mathrm{CaCl}_{2}\end{array}$ & \multicolumn{6}{|c|}{$\mathrm{cmol}$ carga $/ \mathrm{dm}^{3}$} & $\begin{array}{l}\mathrm{V} \\
\%\end{array}$ & $\begin{array}{r}\mathrm{S}-\mathrm{SO} 4 \\
\mathrm{mg} / \mathrm{dm}^{3}\end{array}$ \\
\hline $0-5$ & 10 & 2,3 & 6,0 & 0,12 & 3,30 & 1,84 & 2,02 & 5,3 & 7,3 & 72 & 6 \\
\hline $5-10$ & 6 & 2,2 & 5,0 & 0,14 & 0,61 & 0,50 & 2,77 & 1,3 & 4,0 & 31 & 8 \\
\hline $10-20$ & 5 & 1,7 & 4,4 & 0,11 & 0,33 & 0,30 & 3,08 & 0,7 & 3,8 & 19 & 7 \\
\hline $20-40$ & 4 & 1,5 & 4,2 & 0,20 & 0,29 & 0,11 & 3,81 & 0,6 & 4,4 & 14 & 5 \\
\hline $40-60$ & 3 & 1,3 & 4,2 & 0,05 & 0,22 & 0,07 & 3,08 & 0,3 & 3,4 & 10 & 5 \\
\hline $60-80$ & 3 & 1,2 & 4,2 & 0,04 & 0,22 & 0,06 & 4,01 & 0,3 & 4,3 & 7 & 4 \\
\hline $80-100$ & 3 & 1,0 & 4,3 & 0,09 & 0,31 & 0,06 & 2,63 & 0,5 & 3,1 & 15 & 5 \\
\hline $0-20$ & 3 & 2,1 & 4,6 & 0,06 & 1,00 & 0,60 & 3,40 & 1,7 & 5,1 & 32 & 5 \\
\hline
\end{tabular}

$\left(^{*}\right)$ analises realizadas segundo RAIJ \& QUAGGIO, 1983 
Avaliou-se, no período considerado, o movimento do $\mathrm{S}^{-\mathrm{SO}_{4}}{ }^{-2}$ através do solo estudado. Várias correlações foram estabelecidas entre as doses de gesso agrícola aplicadas, os teores de enxofre nas amostras de terra e os teores do elemento nas amostras de folha.

\section{RESULTADOS E DISCUSSÃO}

Os resultados médios, de nove repetições, dos teores de $\mathrm{S}_{-} \mathrm{SO}_{4}^{-2}\left(\mathrm{mg} / \mathrm{dm}^{3}\right)$ extraidos pelo $\mathrm{NH}_{4} \mathrm{OAc} 0,5 \mathrm{M}$ em HOAc 0,25 $\mathrm{M}$ nas diferentes profundidades, em função das doses de gesso agrícola aplicadas, e aos 6, 12 e 24 meses após a aplicação do produto, encontram-se na TABELA 2. A observação destes, permite concluir, como era esperado, pela grande amplitude de resultados obtidos $\left(3,7\right.$ a $\left.118,7 \mathrm{mg} / \mathrm{dm}^{3} \mathrm{de} \mathrm{S}-\mathrm{SO}_{4}{ }^{-2}\right)$, condição esta fundamental para o estudo que se pretendia desenvolver.

A figura 1, de teores de $\mathrm{S}_{-} \mathrm{SO}_{4}{ }^{-2}$, aos 6 meses após a instalação do ensaio, em função das doses de gesso agrícola aplicadas, e profundidade, permite visualizar melhor a amplitude já comentada, e além disto, verificar de forma banstante clara que o gradiente de teores se deu em função das doses aplicadas.

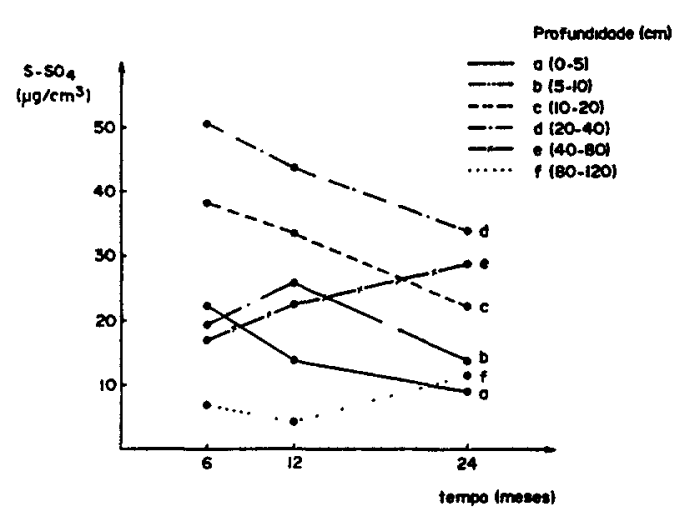

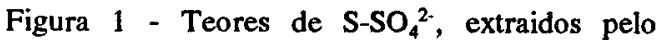
$\mathrm{NH}_{4} \mathrm{OAc} 0,5 \mathrm{M}$ em HOAc 0,25 M, 6 meses após a instalação do ensaio, em função das doses de gesso agrícola aplicadas e profundidade.

Verifica-se que o aumento dos teores de S$\mathrm{SO}_{4}^{-2}$ ocorreu primeiro nas camadas superficiais, porém com o decorrer do tempo o enxofre foi se movimentando para as camadas mais profundas do perfil a ponto de na $3^{\text {a }}$ amostragem a quantidade de $\mathrm{S} \mathrm{SO}_{4}{ }^{-2}$ ter diminuido considera-velmente, em relação à primeira amostragem nas camadas superficiais $\left(94,2\right.$ para $4,3 \mathrm{mg} / \mathrm{dm}^{3}$ de S-SO ${ }_{4}^{-2}$ para a dose 4 t/ha, na camada de 0-5), e aumentado muito nas camadas subsuperficiais (22,2 para 68,8 ug $S / \mathrm{cm}^{3}$ para a dose $4 \mathrm{t} / \mathrm{ha}$, na camada de $80-120$ $\mathrm{cm})$. Uma visão geral da movimentação do $\mathrm{S}_{-} \mathrm{SO}_{4}{ }^{-2}$ no perfil do solo, ao longo do perído considerado no estudo, é obtida na figura 2 , na qual constata-se a diminuição do teor de sıllfato até $40 \mathrm{~cm}$ de profundidade, e o aumento nestes mesmos teores nas camadas $40-80$ e $80-120 \mathrm{~cm}$, em função do tempo decorrido após a aplicação do gesso agrícola (dose de $1 \mathrm{t} / \mathrm{ha}$ ).

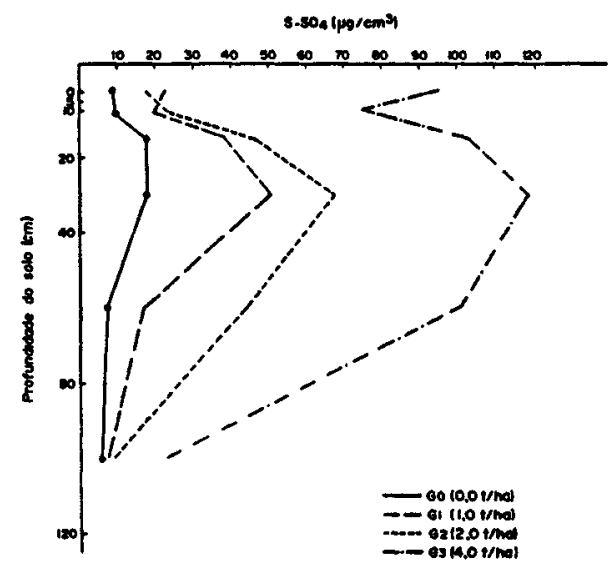

Figura 2 - Teores de $\mathrm{S}_{-} \mathrm{SO}_{4}{ }^{2} \cdot\left(\mathrm{mg} / \mathrm{dm}^{3}\right)$ em lunção do tempo, por profundidade, e para a dose aplicada de 1,0 $t$ de gesso agrícola/ha (extrator $=\mathrm{NH}_{4} \mathrm{OAc} 0,5 \mathrm{M}$ em HOAc 0,25 M).

A TABELA 3 contém as correla-ções entre doses aplicadas de gesso agrícola e teo-res de $S$ $\mathrm{SO}_{4}^{-2}$ nas amostras de terra coletadas nas 3 épocas. Os dados evidenciam que, aos 24 meses após a aplicação do gesso (3a. amostragem) não mais existia efeito do produto até $20 \mathrm{~cm}$ de profundidade, uma vez que as correlações foram não significativas. Este efeito, entretanto, continuava a existir abaixo de $20 \mathrm{~cm}$ de profundidade.

A observação dos dados obtidos para teores de enxofre nas folhas de limão siciliano (TABELA 4), e ainda, das correlações entre doses de gesso agrícola e teores de enxofre nas folhas (TABELA 5), demonstram $o$ efeito de aumento dos teores nas folhas em função das doses crescentes de gesso agrícola nas duas primeiras amostragens $(6 \mathrm{e}$ 
Tabela 2 - Teores de S-SO4 $\left(\mathrm{mg} / \mathrm{dm}^{3}\right)$ nas profundidades de 0-5, 5-10, 10-20, 20-40, 40-80 e 80-120 cm em função das doses de gesso agrícola aos 6, 12 e 24 meses após a aplicação do produto (extrator $=\mathrm{NH}_{4} \mathrm{OAc} 0,5 \mathrm{M} \mathrm{em} \mathrm{HOAc} \mathrm{0,25} \mathrm{M).}$

\begin{tabular}{|c|c|c|c|c|c|c|c|c|c|c|c|c|}
\hline \multirow{3}{*}{$\begin{array}{l}\text { Profun- } \\
\text { didade } \\
\text { (cm) }\end{array}$} & \multicolumn{12}{|c|}{ Gesso - t/ha } \\
\hline & \multicolumn{3}{|c|}{0} & \multicolumn{3}{|c|}{1} & \multicolumn{3}{|c|}{2} & \multicolumn{3}{|c|}{4} \\
\hline & $6(1)$ & 12 & 24 & 6 & 12 & 24 & 6 & 12 & 24 & 6 & 12 & 24 \\
\hline $00-05$ & 8,3 & 8,1 & 5,9 & 22,4 & 13,7 & 9,0 & 17,1 & 15,6 & 7,7 & 94,2 & 68,2 & 4,3 \\
\hline $05-10$ & 9,8 & 7,3 & 9,2 & 19,3 & 25,7 & 13,6 & 23,1 & 19,1 & 11,2 & 74,1 & 63,9 & 11.2 \\
\hline $10-20$ & 17,9 & 11,3 & 18,4 & 38,1 & 33,6 & 22,3 & 46,4 & 36,6 & 27,3 & 103,1 & 59,6 & 28,7 \\
\hline $20-40$ & 17,8 & 11,1 & 29,0 & 50,6 & 43,7 & 34,0 & 67,6 & 43,9 & 46,2 & 118,7 & 74,2 & 53,6 \\
\hline $40-80$ & 7,0 & 3,8 & 6,7 & 17,0 & 22,6 & 28,7 & 43,7 & 34,8 & 38,3 & 100,7 & 94,1 & 69,0 \\
\hline $80-120$ & 5,2 & 3,7 & 4,7 & 7,0 & 4,4 & 11,9 & 8,2 & 8,8 & 18,4 & 22,2 & 43,7 & 68,8 \\
\hline
\end{tabular}

(1) meses após a aplicaçao do gesso agrícola.

12 meses após a aplicação do produto). Na terceira amostragem, entretanto, este efeito já não mais existia.

TABELA 3 - Valores de $r$ das correlações entre doses de gesso agrícola aplicadas e teores de $\mathrm{S}_{-} \mathrm{SO}_{4}^{-2}$ nas amostras de terra (extrator: $\mathrm{NH}_{4} \mathrm{OAc} 0,5 \mathrm{M}$ em HOAc $0,25 \mathrm{M}$ ), por profundidade, aos 6, 12 e 24 meses após a aplicação do produto.

\begin{tabular}{cccc}
\hline & \multicolumn{3}{c}{ amostragem } \\
\cline { 2 - 4 } Prof & $6^{(1)}$ & \multicolumn{1}{c}{12} & 24 \\
\hline $0-5$ & 0,44 & 0,43 & $0,19^{\text {ma }}$ \\
$5-10$ & 0,67 & 0,46 & $0,04^{\mathrm{m}}$ \\
$10-20$ & 0,84 & 0,83 & $0,31^{\mathrm{ms}}$ \\
$20-40$ & 0,87 & 0,83 & 0,59 \\
$40-80$ & 0,90 & 0,78 & 0,85 \\
$80-120$ & 0,72 & 0,67 & 0,73 \\
m(0-20) & 0,78 & 0,56 & $0,22^{\text {na }}$ \\
\hline
\end{tabular}

(1) amostragem após a aplicação de gesso (6,12 e 24 meses)

${ }^{\text {ns }}$ modelo não significativo ao nível de $5 \%$.

quando não assinalado $=$ modelo significativo ao nível citado.

As boas correlações entre teores de sulfato "disponível" no solo e os teores de enxofre nas folhas de limão siciliano nas duas primeiras amostragems (TABELA 6), e nas várias profundidades, poderiam sugerir que a planta esta absorvendo enxofre, em quantidades elevadas, de todas as camadas, inclusive das mais profundas. Entretanto, o fato de na 3a. amostragem ainda existir efeito das doses de gesso agrícola nas profundidades abaixo de $20 \mathrm{~cm}$ (TABELA 3), porém, não mais existir diferenciação nos teores foliares (TABELA 4), fornece evidências de que a planta não extrai quantidades significativas de enxofre das camadas abaixo de $20 \mathrm{~cm}$.

TABELA 4 - Teores de $S$ nas folhas de limão para as 3 amostragens realizadas (6, 12 e 24 meses após a aplicação) em função das doses de gesso agrícola. (média de 9 repetiçōes).

\begin{tabular}{|c|c|c|c|}
\hline \multirow{2}{*}{$\begin{array}{l}\text { Dose } \\
\text { (t/ha) }\end{array}$} & \multicolumn{3}{|c|}{ Amostragem (meses após aplicaçăo do gesso agrícola) } \\
\hline & 6 & 12 & 24 \\
\hline & & \%S & \\
\hline 0 & $0,226 \mathrm{c}^{-}$ & $0,226 \mathrm{c}$ & $0,244 a$ \\
\hline 1 & $0,254 \mathrm{bc}$ & $0,250 \mathrm{c}$ & $0,247 a$ \\
\hline 2 & $0,297 a b$ & $0,276 \mathrm{~b}$ & $0,259 a$ \\
\hline 4 & $0,336 \mathbf{a}$ & 0,328 a & $0,253 a$ \\
\hline
\end{tabular}

* Médias com letras iguais na mesma coluna não diferem entre si ao nível de $5 \%$ pelo teste de Tukey. 
As boas correlações encontradas entre teores no solo, para as várias profundidades, e teores do elemento nas folhas, para as duas primeiras amostragens, se deu provavelmente em virtude do efeito do gesso se pronunciar em todas as profundidades e nas folhas ao mesmo tempo.

TABELA 5 - Correlação: Dose de gesso agrícola e \% $\mathrm{S}$ na folha para as 3 amostragens (6, 12 e 24 meses após a aplicação de gesso agrícola).

\begin{tabular}{ccc}
\hline $\begin{array}{c}\text { Meses após } \\
\text { aplicação }\end{array}$ & $r$ & equação \\
\hline 6 & 0,79 & $\% S=0,0278 . t / \mathrm{ha}+0,229$ \\
12 & 0,90 & $\% \mathrm{~S}=0,0256 . t / \mathrm{ha}+0,225$ \\
24 & 0,25 & $\% \mathrm{~S}=0,0025 . \mathrm{t} / \mathrm{ha}+0,246$
\end{tabular}

TABELA 6 - Valores de $r$ das correlações entre teores de $\mathrm{S}_{-} \mathrm{SO}_{4}{ }^{2-}$ "disponível" no solo (extrator: $\mathrm{NH}_{4} \mathrm{OAc} 0,5 \mathrm{M}$ e HOAc 0,25 M) e teores do elemento nas folhas.

\begin{tabular}{|c|c|c|c|}
\hline \multirow{2}{*}{ Profund. } & \multicolumn{3}{|c|}{ amostragem } \\
\hline & $6^{(1)}$ & 12 & 24 \\
\hline $0-5$ & 0,63 & 0,63 & $0,09^{m}$ \\
\hline $5-10$ & 0,69 & 0,59 & $0,21^{\mathrm{m}}$ \\
\hline $10-20$ & 0,71 & 0,78 & $0,30^{\mathrm{m}}$ \\
\hline $20-40$ & 0,71 & 0,76 & $0,24^{\mathrm{m}}$ \\
\hline $40-80$ & 0,73 & 0,81 & $0,30^{\text {me }}$ \\
\hline $80-120$ & 0,67 & 0,73 & $0,31^{\text {m }}$ \\
\hline$m(0-20)$ & 0,71 & 0,69 & $0,26^{\mathrm{mt}}$ \\
\hline
\end{tabular}

(1)amostragem após a aplicação de gesso (6, 12 e 24 meses)

"modelo não significativo ao nível de 5\% quando não assinalado $=$ modelo significativo ao nível citado.

Os dados indicam, portanto, que 0 estabelecimento dos níveis foliares parece ocorrer em função do $\mathrm{S}_{-} \mathrm{SO}_{4}{ }^{2-}$ da camada superficial, no caso, até $20 \mathrm{~cm}$.

Mesmo não sendo objetivo do trabalho ressalta-se que a análise dos dados de produção de limão (kg de frutos/árvore, média de 6 plantas), em função das doses de gesso agrícola aplicadas (TABELA 7), evidenciam, mesmo diante do aumento dos teores de enxofre nas folhas nas duas primeiras amostragens, não haver resposta de produção para a aplicação do produto. $O$ fato de, mesmo nas parcelas onde se deu a aplicação do gesso agrícola, o teor de enxofre ter se apresentado adequado $(0,20$ a 0,39 segundo MALAVOLTA \& VIOLANTE NETO, 1989) explica a inexistência de aumento de produção.

TABELA 7 - Produção de limão siciliano (kg frutos/árvore) em função das doses de gesso agrícola aplicadas. (Média de 9 repetições, sendo cada repetição a média de 6 plantas).

\begin{tabular}{lccc}
\hline & \multicolumn{3}{c}{ Frutos kg/árvore $^{*}$} \\
\cline { 2 - 4 } $\begin{array}{c}\text { Dose } \\
\text { (t/ha) }\end{array}$ & $1989-$ & 1990 & 1991 \\
\hline 0 & $201,2 \mathrm{a}$ & $223,8 \mathrm{a}$ & $273,2 \mathrm{a}$ \\
1 & $204,1 \mathrm{a}$ & $231,9 \mathrm{a}$ & $275,9 \mathrm{a}$ \\
2 & $200,0 \mathrm{a}$ & $233,1 \mathrm{a}$ & $266,2 \mathrm{a}$ \\
4 & $205,0 \mathrm{a}$ & $235,8 \mathrm{a}$ & $288,4 \mathrm{a}$ \\
\hline C.V. & $10,8 \%$ & $10,8 \%$ & $8,6 \%$ \\
\hline
\end{tabular}

* Médias com letras iguais na vertical não diferem entre si ao nível de $5 \%$ pelo teste de Tukey.

** Ano da colheita

\section{CONCLUSÃO}

Houve evidências que o limão siciliano, enxertado sobre porta enxerto Volkameriano, absorve enxofre, principalmente, da camada de 0 a $20 \mathrm{~cm}$ de profundidade, indicando que esta $\epsilon$ a camada que deve ser considerada para fins de avaliação do teor "disponível" para esta cultura.

\section{REFERÊNCIAS BIBLIOGRÁFICAS}

BARDSLEY, C.E.; KIIMER, V.J. Sulfur supply of soils and crop yields in the southeastern United State. Soil Science Society of America Proceedings, Madison, v.27, n.2, p.197-199, 1963.

BARDSLEY, C.E.; LANCASTER, J.D. Determination of reserve sulfur and soluble sulfates in soils. Soil Science Society American Proceedings, Madison, v.24, p.265-268, 1960. 
BARDSLEY, C.E.; LANCASTER, J.D. Sulfur. In: BLACK, C.A., ed. Methods of soil analysis. Madison: American Society of Agronomy, 1965. pt.2. cap.79, p.1103-1116. (Agronomy, 9).

BLAKEMORE, L.C.; LUDECKE, T.E.; LEAMY, M.L.; METSON, A.J. A study of the pattern of sulphur responses shown by dryland lucerne on some brown-gray earths. New Zealand Journal of Agricultural Research, Wellington, v.12, n.2, p.333-351, 1969.

HARWARD, M.E.; REISENAUER, H.M. Reactions and movement of inorganic soil sulfur. Soil Science. Baltimore, v.101, p.326-335, 1966.

KINJO. T. Natureza das cargas elétricas e capacidade de troca de cátions do solo. In: MARCONI, A. Curso de atualização em fertilidade do solo. Piracicaba: ESALQ, 1980. 23p.
\& A.E. BOARETTO

MALAVOLTA, E.; VIOLANTE NETTO, A. Nutrição mineral, calagem, gessagem e adubaçāo dos citros. Piracicaba: POTAFOS, 1989. 153p.

RAU, B. van. Fertilidade do solo e adubação. Piracicaba: POTAFOS, 1990. 285p.

RAIJ, B. van; QUAGgio, J.A. Métodos de análise de solo para fins de fertilidade. Campinas, Instituto Agronômico, 1983.31p. (IAC. Boletin Técnico, 81).

RAU, B. van; SILVA, N.M. da; BATAGLIA, O.C.: QUAGGIO, J.A.; HIROCE, R.; CANTARELLA, H.; BELLINAZZIJr, R.; DECHEN, A.R.; TRANI, P.E. Recomendaçōes de adubação e calagem para 0 Estado de São Paulo. Campinas: Instituto Agronômico, 1985. 107p. (Boletim Técnico, 100).

Entregue para publicação em 21.06.94 Aceito para publicação em 25.10 .94 